\title{
3D Localization Algorithms for Wireless Sensor Networks
}

\author{
Hiral Patel ${ }^{1}$, Prof. Neha Pandya ${ }^{2}$ \\ ${ }^{1}$ (Computer Science \& Engineering Dept,Parul Institute Of Technology, India) \\ ${ }_{2}^{2}$ (IT Department, ,Parul Institute Of Technology, India)
}

\begin{abstract}
Wireless sensor networks have a great impact on long time monitoring applications (environment monitoring, security surveillance, habitat monitoring etc.) but its potential is yet to be discovered, where it can be deployed in time critical situations when disaster happens. There is a significant gap between existing applications of sensor networks and the requirement of applications supporting rescue operations that involves the catastrophe of human lives. As we are dealing with the human lives here, we can't just rely on the localization schemes that depend upon the connectivity information (range-free) algorithms only. Further, rescue operations are carried out in highly noisy environments, so distance based (range-based) localization algorithms generate high error in distance measurements. An efficient algorithm is needed that can measure the location of the sensor nodes near to the living being or being attached to them in 3-D space with a high accuracy. To achieve such kind of accuracy a combination of both the strategies is required. Further, the algorithms should be efficient and less resource consuming for getting executed on sensor nodes with low processing power. This research proposes an algorithm which incorporates both the range-based and rangefree strategies.
\end{abstract}

Keywords - Wireless sensor network (WSN), Localization.

\section{INTRODUCTION}

This work aims to address the problem of localization in 3D surface wireless sensor networks. First, it reveals the unique hardness in localization on 3D surface in comparison with the well-studied localization problems in 2D and 3D space, and offers useful insight into the necessary conditions to achieve desired localizability. Second, it formulates the localization problem under a practical setting with estimated link distances (between nearby nodes) and nodal height measurements, and introduces a layered approach to promote the localizability of such 3D surface sensor networks.

All the strategies that are employed for the localization in 2-D spaces are violated in 3-D spaces[4,5]. 2-D spaces cannot be directly extended to 3 -D just by increasing one parameter. For example, the 2-D localization completely fails in determining the depth of the river bed and other similar sensor network scenarios where the height comes into play. There are several well-known problems that can be easily solved modeling the sensor network as 2-D but are very complex when modeled as 3-D. Moreover, the main triangulation approach that is employed for the 2-D space doesn't fit for the 3-D.

When we face an unfortunate situation such as an earthquake, hurricane, or similar disasters, wireless sensor nodes prove to be very useful in search and rescue operations. Wireless sensor networks can assist in conducting the rescue operations and can provide search in timely manner. A sensor network mainly consists of anchor nodes as well as the other nodes sensing the data. The anchor nodes are location aware sensor nodes which obtain its location information through some external methods (GPS, TOA[1][2]). The un-localized nodes hear the beacons which are broadcasted by the anchor nodes. Determining the location of these un-localized sensor nodes with respect to the anchor nodes is called localization[3]. The localization algorithms generally possess wave-spreading like characteristics in which the intermediate sensor nodes become location aware and act as a new reference anchor node for the unlocalized neighboring nodes.

The information received from the remote sensors that are several hops far is meaningless until and unless the location from where the data is received is known. For large scale application scenarios where the geographical data is to be known for coarse parameters such as the temperature of an area, the data is aggregated for all the sensor nodes that are located nearby. Further, the location information is important for many location aware sensor network protocols. The positional information is used to partition the network into several clusters for hierarchical routing. For efficient routing the protocols require the information of the location of the sensor nodes.

\section{LOCALIZATION IN WIRELESS SENSOR NETWORKS}

The wireless sensor network is an open research field. Different localization algorithms have been proposed until now. As the sensor networks are application specific it is quite hard to generalize the 
algorithm approaches for localizations that suits to all the different scenarios. All the localization algorithms have been broadly classified into two major categories: range based and range free. Both the strategies employ totally different way of approaches. The range-based method relies on the distance measurements through different received signal strength strategies. Whereas, the range-free method relies on the connectivity information only i.e. the nodes can listen to the beacon from the sensor nodes. The wireless sensor network consists of localized anchor nodes and un-localized sensor nodes. The range free method uses the connectivity information and bound the nodes location to the common overlapped (intersected) area. Different algorithms use different methods to calculate the nodes location information within the bounded region with respect to the anchor nodes.

The range-based method uses the sophisticated hardware, radio signals to estimate the distance between the receiver and the transmitter antennas. Moreover, the range-free approach reuses the wireless communication radio signals to determine the connectivity between neighboring sensors and requires no extra hardware support.

\section{A. Range-Based Localization Schemes: \\ Received Signal Strength (RSS):}

RSS is defined as the power measured by a received signal strength indicator (RSSI) by the receiver. Often, RSS is equivalently reported as measured power, i.e., the squared magnitude of the radio signal strength. We can consider the RSS of low frequency, RF, or other signals. Wireless sensors nodes communicate with neighboring sensors, so the signal strength of the radio can be calculated by each receiver during normal data communication without requiring additional bandwidth or energy requirements. RSS measurements are relatively can be easily embedded in the motes, thought of their expensiveness they are most generally used method for distance calculation[6].

Time Based Methods (TOA \& TDoA):

These methods record the time-of-arrival (ToA) or time-difference-of-arrival (TDoA). The propagation time can be directly translated into distance, based on the known signal propagation speed. These methods can be applied to many different signals, such as RF, acoustic, infrared and ultrasound [9]. TDoA methods are impressively accurate under line-of-sight conditions. But this line-of-sight condition is difficult to meet in some environments. Furthermore, the speed of sound in air varies with air temperature and humidity, which introduce inaccuracy into distance estimation. Acoustic signals also show multi-path propagation effects that may impact the accuracy of signal detection. They rely on complex hardware that is expensive and energy consuming; making it less suitable for sensor networks where the scalability is high of the lifetime of the network is expected to be more.

\section{Angle Of Arrival (AOA):}

AoA estimates the angle at which signals are received and use simple geometric relationships to calculate node positions. Generally, AoA techniques provide more accurate localization result than RSSI based techniques but the cost of hardware of very high in AoA. By providing information about the direction to neighboring sensors nodes rather than the distance to adjacent sensors nodes, AOA measurements provide localization information complementary to the TOA and RSS measurements. Sophisticated direction aware antennas with high synchronization clocks are need in this method [10,11]. Similar to AOA, TOA and TDOA estimates require additional hardware too expensive to be used in high scalable sensor networks.

\section{B. Range-Free Localization Schemes: Centroid Algorithm:}

Anchors send their location information to neighbors that keep an account of all received beacons. Using the averaged out information, simple centroid model is applied for estimation the listening nodes location. This protocol is referred as the Centroid algorithm. The following are the steps of the localization:

$\checkmark$ Beacon node broadcasts their position.

$\checkmark$ Sensor node listens for beacons from anchors nodes and if they are able to hear the beacons this means they are under the ranging area covered by these anchor nodes.

$\checkmark$ Sensor node computes its position by averaging out all the beacon node locations.

\section{DV-HOP:}

DV-HOP assumes that a network consisting of identical sensing nodes and beacon nodes [7]. Instead of going for the single hop broadcast, anchors flood their locations all over the sensor network. As the information propagates over each hop an increment counter each time increments the hop count value. Senor nodes calculate their position based on the received beacon locations, average distance per hop, the hop count from the corresponding anchor; the working of this strategy is quite similar to existing 
distance vector routing. One anchor node broadcasts a beacon to all over the network containing the beacon location with a hop count value initially set to one.

Multi-Dimensional Scaling (Mds):

The multi-dimensional scaling is another type of range-free method. In a large scale sensor networks, Multi-Dimensional Scaling (MDS) only uses the connectivity information. This process has three steps: Roughly estimate the distance between all the sensor node pairs. Apply the MDS to derive locations fitted roughly to the estimated distances. Optimize by taking the known sensor node locations.

APIT (Approximate Point in Triangulation):

It is the one of the backbone strategy employed by most of the range-free methods [8]. In this approach the whole sensor network environment is divided into triangles. The triangles are formed by choosing the combinations of the anchor nodes. If the sensor node is within the triangular area formed by the three anchor nodes the possible area of presence of the sensor node constraints up to the triangular area only.. This process goes until all the possible sets of forming the triangles are exhausted, or the desired accuracy is reached.

\section{PROPOSED 3D Localization Algorithm}

The following steps are performed for calculation of 3-D localization:

$\checkmark$ The whole area is divided into grids. The interspacing between the grids is less than the range of the anchor nodes.

$\checkmark$ A beacon node covers a block of cubes that are within its radio range. The cube size is such that it covers the largest area in the sphere formed by the radio range.

$\checkmark$ The distance between the un-localized sensor node and the beacon nodes is calculated using different RSS techniques.

$\checkmark$ A list is maintained by each un-localized node that contains the number of beacon nodes and the distance form each beacon node.

$\checkmark$ The common cubical intersection areas of all the beacon nodes is found reducing the location possibilities.

$\checkmark$ The common intersection area gives the bounded $\beta$ values (max, min).The bounded values specifies the maximum and minimum values of co-ordinates. The nodes position should be within this rectangular region.

$\checkmark \quad$ Check whether the beacon nodes $(\geq 3)$ are forming a plane i.e. they are not on same line.

$\checkmark$ Calculate the location (x, y, z) of un-localized nodes taking the distances into account.

$\checkmark$ Check the whether the calculated values within bounding region. All the values outside the region are discarded.

$\checkmark$ Change the status of newly localized node to new beacon nodes for further calculation of other nodes.

\section{The bounded area is explained below (Range-Free Approach):}

Instead of considering a spherical area around a sensor node we consider the sensor node covers a cubical area. All the nodes within the cubical area can listen to anchor node other outside it are not considered to be covered by this anchor node.

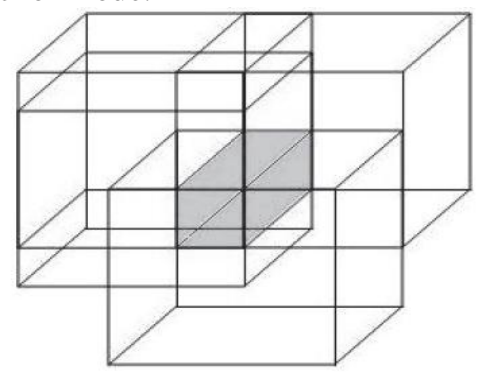

Figure1: Intersection of bounding boxes, shaded region is the common bounded region.

Each cube representing the bounding area can be represented by the min \& max values of its coordinates. The radio range covered by the beacon node $\mathrm{P}(\mathrm{x}, \mathrm{y}, \mathrm{z})$ varies between $\left[x_{\min }, y_{\min }, z_{\min }\right]$ and $\left[x_{\max }, y_{\max }, z_{\max }\right.$ ] represents the bounded range $\beta$ of a node.

$$
\text { Where, }\left\{\begin{array} { l } 
{ x _ { \operatorname { m i n } } = x - r + \partial } \\
{ y _ { \operatorname { m i n } } = y - r + \partial } \\
{ z _ { \operatorname { m i n } } = z - r + \partial }
\end{array} \quad \left\{\begin{array}{l}
x_{\max }=x+r-\partial \\
y_{\max }=y+r-\partial \\
z_{\max }=z+r-\partial
\end{array}\right.\right.
$$

$\partial$ corresponds to the error due to irregular radio pattern.

$r$ average radio range of the beacon, 


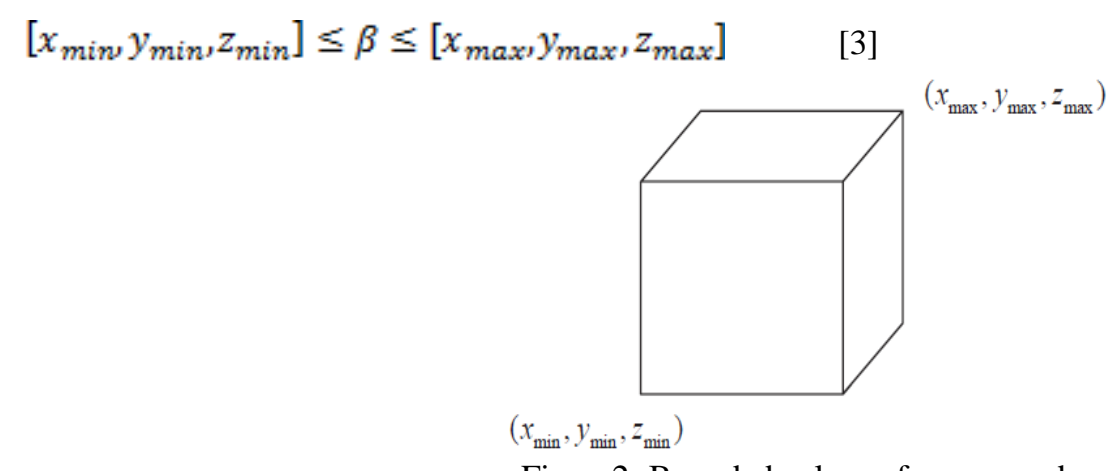

Figure2: Bounded values of sensor nodes

The intersection of the two cubical areas is done to reduce the area where the un-localized node is likely to be. As, more number of cubical areas start to merge the common area of intersection starts to reduce. The new rectangular (sometimes square) area that emerges after multiple intersections of beacon nodes represents the bounded area. For example intersection of two bounding cubes;

$\beta_{\text {new }}=\beta_{1} I \beta_{2}$

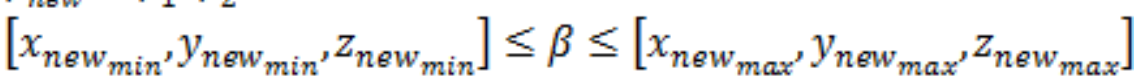

Where, $\left\{\begin{array}{l}x_{n e w_{\min }}=\left|x_{2_{\min }}-x_{1_{\min }}\right| \\ y_{n e w_{\min }}=\left|y_{2_{\min }}-y_{1_{\min }}\right| \\ z_{n e w_{\min }}=\left|z_{2_{\min }}-z_{1_{\min }}\right|\end{array} \quad\left\{\begin{array}{l}x_{n e w_{\max }}=\left|x_{2_{\max }}-x_{1_{\max }}\right| \\ y_{n e w_{\max }}=\left|y_{2_{\max }}-y_{1_{\max }}\right| \\ z_{n e w_{\max }}=\left|z_{2_{\max }}-z_{1_{\max }}\right|\end{array}\right.\right.$

Similarly, intersection of multiple boundaries can be done to lower the number of positions where a sensor node can be present.

$\beta_{\text {new }}=\beta_{1} I \beta_{2} I \ldots \ldots \ldots \beta_{n} I$

Where, $\mathrm{n}$ is the number of beacon nodes covering a un-localized node.

The method falls under the category of range-free as it only uses the connectivity information i.e. whether it is able to send \& receive the beacon nodes from the respective beacon nodes.

D. Calculating from known distances (Range-Based):

A set of three anchor nodes are selected at a time. These three anchor nodes form a equation of a plane.

The standard equation of a plane in 3 dimensional spaces is

$\mathrm{Qx}+\mathrm{Ry}+\mathrm{Sz}+\mathrm{D}=0$

Here in equation, Q, R, S correspond to the anchor nodes \& D corresponds to the sensor node with unknown location information. The normal to the plane is the vector $(\mathrm{Q}, \mathrm{R}, \mathrm{S})$.

Given three points in space $\mathrm{Q}(\mathrm{x} 1, \mathrm{y} 1, \mathrm{z} 1), \mathrm{S}(\mathrm{x} 2, \mathrm{y} 2, \mathrm{z} 2), \mathrm{S}(\mathrm{x} 3, \mathrm{y} 3, \mathrm{z} 3)$ the equation of the plane through these points is given by the following determinants.

$\begin{array}{ll}\mathbf{Q}=\left(\begin{array}{lll}1 & y_{1} & z_{1} \\ 1 & y_{2} & z_{2} \\ 1 & y_{3} & z_{3}\end{array}\right) & \mathbf{S}=\left(\begin{array}{lll}x_{1} & y_{1} & 1 \\ x_{2} & y_{2} & 1 \\ x_{3} & y_{3} & 1\end{array}\right) \\ \mathbf{R}=\left(\begin{array}{lll}x_{1} & 1 & z_{1} \\ x_{2} & 1 & z_{2} \\ x_{3} & 1 & z_{3}\end{array}\right) & \mathbf{D}=-\left(\begin{array}{lll}x_{1} & y_{1} & z_{1} \\ x_{2} & y_{2} & z_{2} \\ x_{3} & y_{3} & z_{3}\end{array}\right)\end{array}$

Expanding the above gives :

$Q=y_{1}\left(z_{2}-z_{3}\right)+y_{2}\left(z_{3}-z_{1}\right)+y_{3}\left(z_{1}-z_{2}\right)$

$R=z_{1}\left(x_{2}-x_{3}\right)+z_{2}\left(x_{3}-x_{1}\right)+z_{3}\left(x_{1}-x_{2}\right)$

$S=x_{1}\left(y_{2}-y_{3}\right)+x_{2}\left(y_{3}-y_{1}\right)+z_{3}\left(y_{1}-y_{2}\right)$

$\mathrm{D}=-\left[X_{1}\left(Y_{2} Z_{3}-Y_{3} Z_{2}\right)+X_{2}\left(Y_{3} Z_{1}-Y_{1} Z_{3}\right)+X_{3}\left(Y_{1} Z_{2}-Y_{2} Z_{1}\right)\right]$

The sign of, $\mathrm{s}=\mathrm{Qx}+\mathrm{Ry}+\mathrm{Sz}+\mathrm{D}$ determines which side the point $(x, y, z) \quad$ lies with respect to the plane. If $\mathrm{s}$ $>0$ then the point lies on the same side as the normal $(\mathrm{Q}, \mathrm{R}, \mathrm{S})$. If $\mathrm{s}<0$ then it lies on the opposite side, if $\mathrm{s}=0$ then the point $(x, y, z)$ lies on the plane.

For a plane $\mathrm{Qx}+\mathrm{Ry}+\mathrm{Sz}+\mathrm{D}=0$ and a point $P(x, y, z)$ not necessarily lying on the plane, the shortest distance from $\mathrm{P}$ to the plane is.

Distance $=\frac{\left|Q x_{1}+R y_{1}+S z_{1}+d\right|}{\sqrt{Q^{2}+R^{2}+S^{2}}}$ 


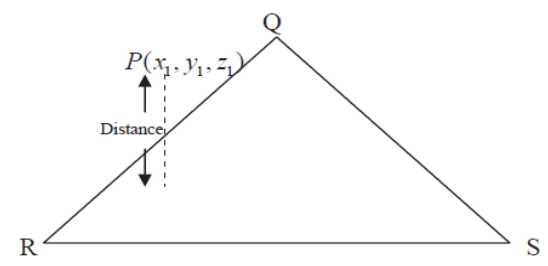

Figure3: Distance of point $\mathrm{P}$ from a plane

It follows that if Distance $=0$ in equation 24 then $\mathrm{P}$ lies in the same plane. However, in real life environmental conditions where there is interferencing noise the measuring and calculating errors might change the distance a little bit. As a result $\mathrm{P}$ is considered lying within the same plane if it satisfies the constraint: The distances are measured by using different received signal strength (RSS) strategies. At least three beacon nodes are required to calculate the location of unknown sensor node.

$\left(x-x_{i}\right)^{2}+\left(y-y_{i}\right)^{2}+\left(z-z_{i}\right)^{2}=p_{i}^{2} \quad \mathrm{i}=1,2,3 \ldots \ldots$

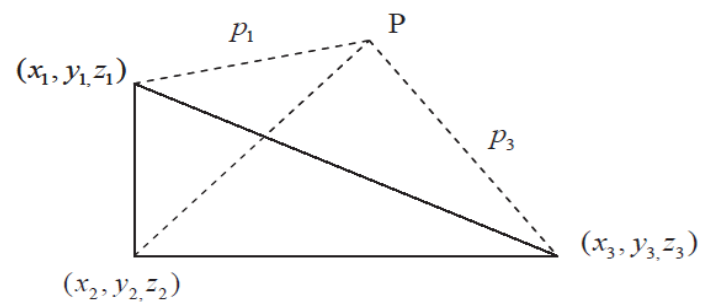

Figure4: unknown coordinates of $\mathrm{P}: p_{1}, p_{2}, p_{3}$ are calculated distances using RSS strategies.

$\left(x-x_{1}\right)^{2}+\left(y-y_{1}\right)^{2}+\left(z-z_{1}\right)^{2}-p_{1}^{2}=0$

$\left(x-x_{2}\right)^{2}+\left(y-y_{2}\right)^{2}+\left(z-z_{2}\right)^{2}-p_{2}^{2}=0$

$\left(x-x_{3}\right)^{2}+\left(y-y_{3}\right)^{2}+\left(z-z_{3}\right)^{2}-p_{3}^{2}=0$

In General, $\left(x-x_{n}\right)^{2}+\left(y-y_{n}\right)^{2}+\left(z-z_{n}\right)^{2}-d_{n}{ }^{2}=0$, Where $\left(x_{n}, y_{n}, z_{n}\right)$ corresponds the location of the sensor nodes.

\section{Simulation RESULTS}

The algorithm proposed here, as all the geometric approaches of the localization problem, requires a large number of sensor nodes and location aware beacons. All the simulations related to thesis is done in MATLAB. MATLAB simulator is selected as it easily handles the matrix calculations very effectively \& all the stress of the work can be given to the actual work rather than going on programming details.

Effect of Radio Range:

\begin{tabular}{|l|l|l|l|}
\hline $\begin{array}{l}\text { No of nodes } \\
\text { per layer }\end{array}$ & $\begin{array}{l}\text { Radio } \\
\text { Range }(\mathbf{m})\end{array}$ & $\begin{array}{l}\text { \% Success in } \\
\text { proposed method }\end{array}$ & $\begin{array}{l}\text { Total } \\
\text { time(sec) }\end{array}$ \\
\hline 100 & 4 & 86.9 & 3.1720 \\
\hline 100 & 5 & 85.5 & 3.0470 \\
\hline 100 & 6 & 87.5 & 3.3750 \\
\hline 100 & 8 & 95.6 & 4.3440 \\
\hline
\end{tabular}

Table1: Effect of Radio Range

The performance is observed w.r.t. the variations in radio range. Table gives the simulated results. The above simulation is carried out taking the inter layer separation $3 \mathrm{~m}$. As the proposed utilizes both the range-free and range-based strategies there is not much performance change in it.

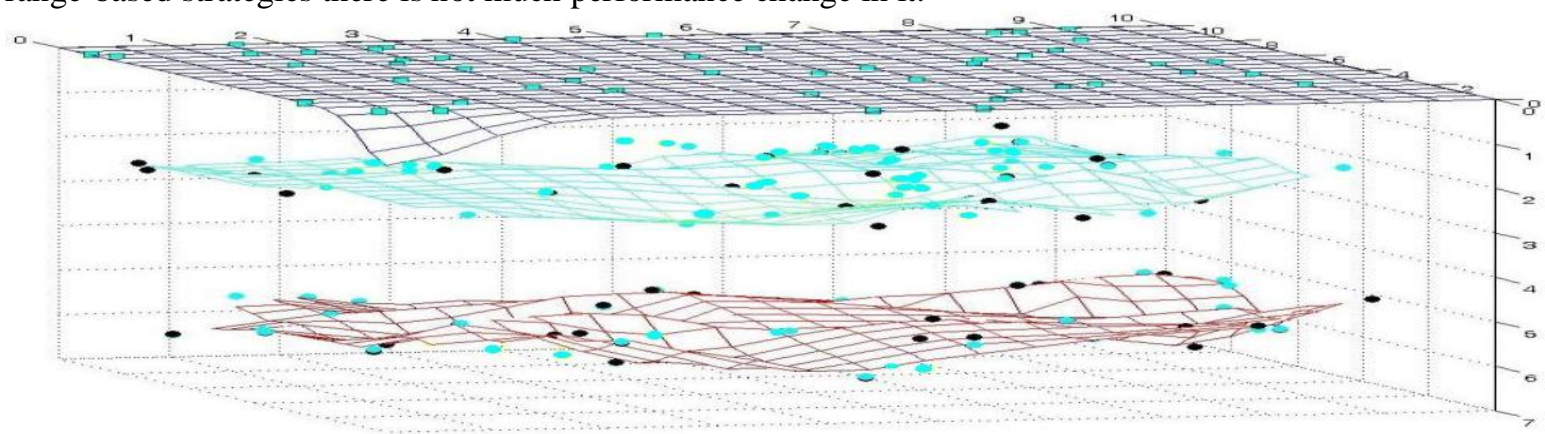

Figure5: Simulation done taking 150 nodes with radio range $5 \mathrm{~m}$ 
The simulation time taken increases irrespective of the number of sensor nodes as for single layer information of location from multiple layers of beacon nodes are processed.

In this way, the noise here is considered to be Gaussian Noise with mean $\mu=0$ and standard Deviation $\sigma$. Here, effect of noise are not shown but from the results we can see that the noise has direct impact on estimation accuracy. As the noise value increases the success rate of the proposed scheme varies between 55\% $95 \%$. Though the success rate drops but still it gives better results than other methods.

\section{CONCLUSION AND FUTURE WORK}

The literature survey work has shown that an optimal algorithm has not been defined yet, that employ both the strategies (range-free \& range-based), and thus the development of a new algorithm has to be founded on the specificities of the situations, taking into account the size of the network, as well as the deployment methods and the expected results.

Localization algorithms should be designed to achieve low variance as well as low bias as far as possible; at the same time, they need to be scalable to very large network sizes without dramatically increasing energy consumption or computational requirements.

Moreover, with one of the ideas proposed, the Bounding cube. This leads to major developments that have been proposed in this research which will improves significantly to increase the localization accuracy. They do not require much more computational costs and perfectly match the distributed algorithm's requirements.

We have proposed and demonstrated an algorithm that successfully localizes nodes in a sensor network with noisy distance measurements. The equations for the proposed algorithm were carried out in MATLAB. Simulations showed the relationship between noise and ability of a network to localize itself, at highly noisy environments. The performance stays above 50\% i.e. half of the available nodes can be localized with good approximation.

\section{REFERENCES}

[1] J. Hightower, C. Vakili, G. Borriello, and R. Want, "Design and calibration of the spotonad-hoc location sensing system," CSE Techniuqe Report, University of Washington,August 2001.

[2] R. L. Moses, D. Krishnamurthy, and R. Patterson, "An auto-calibration method for unattended ground sensors," in Proc. ICASSP, vol. 3, May 2002, pp. 2941-2944.

[3] Neal Patwari, Alfred O. Hero, III, Matt Perkins, Neiyer S. Correal, Robert J. O'Dea,"Relative Location Estimation in Wireless Sensor Networks', 2003.

[4] S. M. Nazrul Alam, Zygmunt J. Haas, "Topology Control and Network Lifetime inThree-Dimensional Wireless Sensor Networks",2006

[5] I.F.Akyildiz, D. Pompili and T. Melodia, "Underwater Acoustic Sensor Networks:Research Challenges", Ad Hoc Networks Journal, (Elsevier), March 2005.

[6] Neal Patwari, Joshua N. Ash, Spyros Kyperountas, Alfred O. Hero III, Randolph L.Moses, and Neiyer S. Correal, "Locating the Nodes :Cooperative localization in wireless sensor networks", 2005

[7] D. Niculescu and B. Nath, "DV Based Positioning in Ad hoc Networks", In Journal of Telecommunication Systems, 2003.

[8] T. He, C. Huang, B. Blum, J. Stankovic, and T. Abdelzaher, "Rangefree localization schemes in large scale sensor networks," in Proc. ACM/IEEE 9th Annu. Int. Conf. Mobile Computing and Networking (MobiCom'03), 2003.

[9] Andreas Savvides, Chih-Chieh Han, and Mani B. Strivastava, "Dynamic fine-grained localization in Ad-Hoc networks of sensors", 7-th annual international conference on Mobile computing and networking (MobiCom) 2001, pp. 166-179, July 16 - $21,2001$.

[10] D. Niculescu and B. Nath, “Ad Hoc Positioning System (APS) using AoA”, INFOCOM’ 03, San Francisco, CA, 2003.

[11] Niculescu and Badri Nath, "Ad Hoc Positioning System (APS) Using AOA Dragos",2003. 